\title{
Nonlocal Flow of Control and Kleene Algebra with Tests
}

\author{
Dexter Kozen \\ Department of Computer Science \\ Cornell University \\ Ithaca, New York 14853-7501, USA
}

\begin{abstract}
Kleene algebra with tests (KAT) is an equational system for program verification that combines Kleene algebra (KA), or the algebra of regular expressions, with Boolean algebra. It can model basic programming and verification constructs such as conditional tests, while loops, and Hoare triples, thus providing a relatively simple equational approach to program equivalence and partial correctness. In this paper we show how KAT can be used to give a rigorous equational treatment of control constructs involving nonlocal transfer of control such as unconditional jumps, loop statements with multi-level breaks, and exception handlers. We develop a compositional semantics and a complete equational axiomatization. The approach has some novel technical features, including a treatment of multi-level break statements that is reminiscent of de Bruijn indices in the variable-free lambda calculus. We illustrate the use of the system by giving a purely calculational proof that every deterministic flowchart is equivalent to a loop program with multi-level breaks.
\end{abstract}

\section{Introduction}

Kleene algebra with tests (KAT) is an equational system for program verification that combines Kleene algebra (KA), or the algebra of regular expressions, with Boolean algebra. It can model basic programming and verification constructs such as conditional tests, while loops, and Hoare triples, thus providing a relatively simple equational approach to program equivalence and partial correctness. It has been applied successfully in numerous low-level verification tasks involving communication protocols, source-to-source program transformations, concurrency control, com- piler optimization, and dataflow analysis $[1,3,6,7$, $15,16,18,21,23]$. The formalism allows a clean separation between first-order reasoning to establish basic premises, which may take properties of the domain of computation into account, and program manipulation, which is chiefly propositional.

There is an extensive model theory with various classes of language, relational, trace, and matrix models. All these classes share the same equational theory, and KAT is deductively complete for this theory. The relational models are of particular interest in programming language semantics. KAT subsumes propositional Hoare logic (PHL) and is deductively complete for all relationally valid Hoare-style rules, whereas PHL is not [19]. The equational theory is PSPACE-complete, the same as KA and PHL [8].

There is a coalgebraic theory of KAT based on a generalization of classical automata theory to include Booleans [20,22]. The generalized automata are known as automata with tests or automata on guarded strings. An ordinary automaton with $\varepsilon$-transitions is an automaton with tests over the two-element Boolean algebra 2. This theory provides a more rigorous algebraic foundation for classical program schematology that allows simpler and more rigorous equivalence and inequivalence proofs $[1,25]$. Informal combinatorial arguments and surgery on graph models are replaced with purely calculational equational proofs that are more amenable to automation.

Although KAT is particularly well suited for reasoning about while programs (programs constructed from atomic actions and tests with sequential composition, conditionals, and while loops), there is a misconception that it is less well suited for dealing with more general control constructs involving nonlocal transfer of control such as goto statements, loop statements with multi-level breaks, and exception handlers. These constructs are typically handled with higher-order meth- 
ods involving continuation passing.

In this paper, we attempt to dispel this misconception. We present a compositional semantics for goto, loop, and multi-level break statements and a complete equational axiomatization. The approach has some novel technical features, including an interesting treatment of the break $n$ statement that is reminiscent of de Bruijn indices in the variable-free lambda calculus [10]. We illustrate the use of the system by giving a purely calculational proof of the classical folklore result that every deterministic flowchart is equivalent to a loop program with multi-level breaks.

\section{Definitions}

\subsection{Kleene Algebra}

Kleene algebra (KA) is the algebra of regular expressions $[9,13]$. The axiomatization here is from [17]. A Kleene algebra is a structure $\left(K,+, \cdot{ }^{*}, 0,1\right)$ such that $K$ is an idempotent semiring under $+, \cdot, 0$, and 1 and satisfies the axioms

$$
\begin{array}{ll}
1+p p^{*} \leq p^{*} & q+p r \leq r \Rightarrow p^{*} q \leq r \\
1+p^{*} p \leq p^{*} & q+r p \leq r \Rightarrow q p^{*} \leq r
\end{array}
$$

for $*$. There is a natural partial order $p \leq q \stackrel{\text { def }}{\Longleftrightarrow} p+q=$ $q$.

Standard models include the family of regular sets over a finite alphabet, the family of binary relations on a set, and the family of $n \times n$ matrices over another Kleene algebra. Other more unusual interpretations include the min,+ algebra, also known as the tropical semiring, used in shortest path algorithms, and models consisting of convex polyhedra used in computational geometry [11].

The completeness result of [17] says that the algebra of regular sets of strings over a finite alphabet $\Sigma$ is the free Kleene algebra on generators $\Sigma$. The axioms are also complete for the equational theory of relational models.

\subsection{Kleene Algebra with Tests}

A Kleene algebra with tests (KAT) [18] consists of a Kleene algebra $K$ with an embedded Boolean algebra $B$ such that the semiring structure on $B$ is a subalgebra of the semiring structure on $K$. Elements of $B$ are called tests. The Boolean negation operator is defined only on tests.

Like KA, KAT has language and relational models and is deductively complete over these interpretations [24]. The chief language-theoretic models are the regular sets of guarded strings over alphabets $\Sigma$ and $T$ of primitive action and test symbols, respectively (see Section 2.3). This is the free KAT on generators $\Sigma, T$. The set of guarded strings represented by a KAT expression $e$ is denoted $\mathrm{GS}(e)$.

KAT can code elementary programming constructs and Hoare partial correctness assertions and subsumes propositional Hoare logic (PHL). It is deductively complete over relational models, whereas PHL is not. Moreover, KAT is no more difficult to decide, as PHL, KA, and KAT are all PSPACE-complete.

For KAT expressions $e, e^{\prime}$, we write $e \leq e^{\prime}$ or $e=e^{\prime}$ if the relation holds in the free KAT on generators $\Sigma, T$; that is, if it is a consequence of the axioms of KAT.

See $[17,18,19]$ for a more detailed introduction.

\subsection{Guarded Strings}

Guarded strings were introduced in [12]. Let $\Sigma$ be a finite set of action symbols and $T$ a finite set of test symbols disjoint from $\Sigma$. The symbols $T$ generate a free Boolean algebra $B$; elements of $B$ are called tests. An atom is a minimal nonzero element of $B$. The set of atoms is denoted At. The elements of At can be regarded either as conjunctions of literals of $T$ (elements of $T$ or their negations) or as truth assignments to $T$. We write $p, q, p_{0}, \ldots$ for elements of $\Sigma$ and $\alpha, \beta, \alpha_{0}, \ldots$ for elements of At. A guarded string is a finite alternating sequence of atoms and actions, beginning and ending with an atom; that is, an element of $(\text { At } \cdot \Sigma)^{*}$. At. We will also refer to infinite guarded strings, which are members of $(\text { At } \cdot \Sigma)^{\omega}$, but will always qualify with the adjective "infinite" when doing so.

\subsection{Automata with Tests}

Automata with tests, also known as automata on guarded strings, were introduced in [20]. They are the automata-theoretic counterpart to Kleene algebra with tests (KAT). In the formalism of [20], they have two types of transitions, action transitions and test transitions, and operate over guarded strings. An ordinary automaton with $\varepsilon$-transitions is just an automaton with 
tests over the two-element Boolean algebra 2. Many of the constructions of ordinary finite-state automata, such as determinization and state minimization, generalize readily to automata with tests. In particular, there is a version of Kleene's theorem showing that these automata are equivalent in expressive power to expressions in the language of KAT.

Deterministic flowcharts correspond to a limited class of automata called strictly deterministic [25]. Intuitively, a strictly deterministic automaton operates by starting in its start state and scanning a sequence of atoms, which we can view as provided by an external agent. For each atom in succession, the automaton responds deterministically either by emitting an action symbol and moving to a new state, or by simply halting, according to its transition function.

Formally, a strictly deterministic automaton over $\Sigma$ and $T$ is a tuple

$$
M=(Q, \delta, \text { start })
$$

where $Q$ is a finite set of states, start $\in Q$ is the start state, and $\delta$ is a transition function

$$
\delta: Q \rightarrow \text { At } \rightarrow(\Sigma \times Q)+\{\text { halt }\}
$$

where + denotes disjoint (marked) union. The element halt is not a state, but a universal constant used by an automaton to signal halting.

Given a state $s$ and an infinite sequence of atoms $\sigma$, there is a unique finite or infinite guarded string gs $(s)(\sigma)$ obtained by running the automaton starting in state $s$. Formally, the map

$$
\text { gs }: Q \rightarrow A t^{\omega} \rightarrow(\mathrm{At} \cdot \Sigma)^{*} \cdot \mathrm{At}+(\mathrm{At} \cdot \Sigma)^{\omega}
$$

is defined coinductively as follows:

$$
\operatorname{gs}(s)(\alpha \sigma) \stackrel{\text { def }}{=} \begin{cases}\alpha \cdot p \cdot \operatorname{gs}(t)(\sigma), & \text { if } \delta(s)(\alpha)=(p, t) \\ \alpha, & \text { if } \delta(s)(\alpha)=\text { halt. }\end{cases}
$$

This determines $\operatorname{gs}(s)(\sigma)$ uniquely for all $s \in Q$ and $\sigma \in A^{\omega}$.

The set of (finite) guarded strings represented by the automaton $M$ is

$$
\mathrm{GS}(M) \stackrel{\text { def }}{=}\left\{\mathrm{gs}(\text { start })(\sigma) \mid \sigma \in \mathrm{At}^{\omega}\right\} \cap(\text { At } \cdot \Sigma)^{*} \cdot \text { At. }
$$

Two automata are considered equivalent if they represent the same set of finite guarded strings.

\subsection{Programming Constructs}

Deterministic while programs are formed inductively from sequential composition $(p ; q)$, conditional test (if $b$ then $p$ else $q$ ), and while loops (while $b$ do $p$ ), where $b$ is a test and $p, q$ are programs. We also include instructions skip (do nothing) and fail (looping or abnormal termination), although these constructs are redundant, being semantically equivalent to while false do $p$ and while true do skip, respectively. We do not include a halt instruction; a program terminates normally by falling off the end.

Every while program can be converted to an equivalent strictly deterministic automaton. One first converts the programs to a KAT term using the standard translation

$$
\begin{array}{rlrl}
p ; q & =p q & \text { skip } & =1 \\
\text { if } b \text { then } p \text { else } q & =b p+\bar{b} q & \text { fail }=0 \\
\text { while } b \text { do } p & =(b p)^{*} \bar{b}, & &
\end{array}
$$

then applies Kleene's theorem for KAT to yield an automaton with tests, which can then be converted to the form of Section 2.4. This construction is given in [25]. An example is shown in Fig. 1. In the strictly deterministic automaton shown in that figure, an edge from $s$ to $t$ labeled $\alpha p$ denotes the transition $\delta(s)(\alpha)=(p, t)$. The converse is false: there is a strictly determinis-

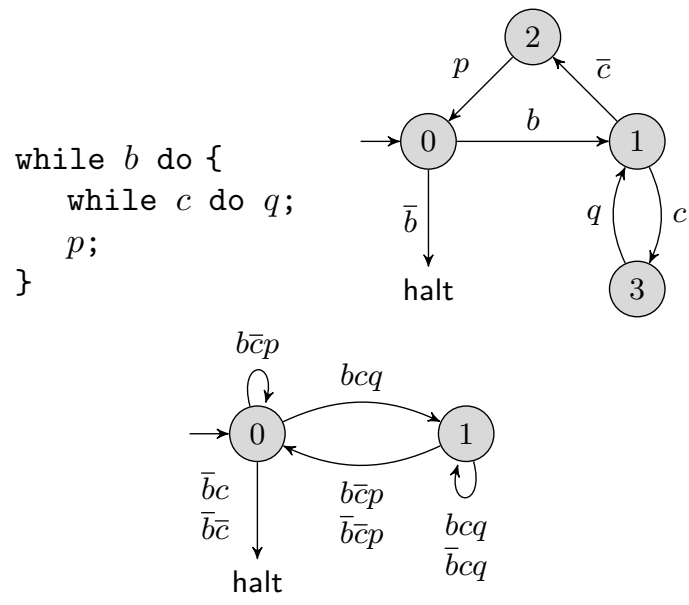

Figure 1: A while program and its corresponding deterministic flowchart and strictly deterministic automaton

tic automaton equivalent to no while program $[2,14]$ (see also [25], in which a three-state counterexample is given). 
In addition to the usual while program constructs, we consider the following constructs:

$$
\begin{array}{ll}
\text { loop } p & \text { goto } \ell \\
\text { break } n, n \geq 1 & \ell: p
\end{array}
$$

Intuitively, the loop construct causes repeated execution of its body $p$. If $p$ terminates normally, then control is transferred back to the beginning of $p$. This construct can be used in conjunction with the nonlocal breaks break $n, n \geq 1$. Intuitively, a break $n$ instruction transfers control to the location immediately following the $n$th loop within whose scope the instruction occurs, counting from the innermost. The statement break is short for break 1 . We also consider the unconditional jump goto $\ell$, which transfers control to a labeled program $\ell: p$ if it exists, or fails if not.

The numbers $n$ in the instructions break $n$ are called de Bruijn indices in recognition of their similarity to the construct of the same name in the variable-free lambda calculus [10]. The index 0 denotes the continuation that corresponds to "falling off the end of the program" and is also considered a de Bruijn index. The de Bruijn indices along with the statement labels $\ell$ (which are assumed to be disjoint from the de Bruijn indices) are collectively called continuation labels.

\section{Semantics}

Programs $p$ are interpreted by a translation to KAT expressions. This is a two-step process. In the first step, we identify a family of expressions $R_{\alpha}(p)$, one for each continuation label $\alpha$. Intuitively, $R_{0}(p)$ is a KAT expression representing all valid halting computations that do not encounter a break or goto instruction. The expression $R_{n}(p)$ for $n \geq 1$ represents the set of computations leading to a break $n$ (or more accurately, to any statement that has the same effect as a break $n$ occurring at the outermost level). The expression $R_{\ell}(p)$ represents the set of computations leading to goto $\ell$. The definitions are compositional but interdependent, thus must be defined by mutual induction.

In the second step, we define expressions $R_{\ell \alpha}(p)$ for each statement label $\ell$ and continuation label $\alpha$. This represents the set of computations in $p$ leading to the continuation $\alpha$ starting at the label $\ell$. These pieces are then composed using a matrix construction to give a single expression denoting the set of valid computations of the program.
For convenience, define

$$
s(\alpha)= \begin{cases}\ell, & \text { if } \alpha=\ell, \\ n+1, & \text { if } \alpha=n .\end{cases}
$$

For a test or primitive action $p$, let

$$
R_{\alpha}(p) \stackrel{\text { def }}{=} \begin{cases}p, & \text { if } \alpha=0 \\ 0, & \text { otherwise }\end{cases}
$$

For the nonlocal atomic constructs,

$$
\begin{aligned}
& R_{\alpha}(\text { break } n) \stackrel{\text { def }}{=} \begin{cases}1, & \text { if } \alpha=n, \\
0, & \text { otherwise },\end{cases} \\
& R_{\alpha}(\text { goto } \ell) \stackrel{\text { def }}{=} \begin{cases}1, & \text { if } \alpha=\ell, \\
0, & \text { otherwise. }\end{cases}
\end{aligned}
$$

For nonatomic programs,

$$
\begin{aligned}
& R_{\alpha}(p+q) \stackrel{\text { def }}{=} R_{\alpha}(p)+R_{\alpha}(q) \\
& R_{\alpha}(p q) \stackrel{\text { def }}{=} \begin{cases}R_{0}(p) \cdot R_{0}(q), & \text { if } \alpha=0, \\
R_{\alpha}(p)+R_{0}(p) \cdot R_{\alpha}(q), & \text { otherwise }\end{cases} \\
& R_{\alpha}\left(p^{*}\right) \stackrel{\text { def }}{=} R_{0}(p)^{*} \cdot R_{\alpha}(p)+R_{\alpha}(1) \\
& = \begin{cases}R_{0}(p)^{*}, & \text { if } \alpha=0, \\
R_{0}(p)^{*} \cdot R_{\alpha}(p), & \text { otherwise, }\end{cases} \\
& R_{\alpha}(\text { loop } p) \stackrel{\text { def }}{=} R_{s(\alpha)}\left(p^{*}\right)=R_{0}(p)^{*} \cdot R_{s(\alpha)}(p) \text {. }
\end{aligned}
$$

Define $p \equiv q$ if $R_{\alpha}(p)=R_{\alpha}(q)$ for all continuation labels $\alpha$. Since the meaning of programs will depend only on the $R_{\alpha}$, two $\equiv$-equivalent programs can be substituted for each other.

Theorem 3.1 The relation $\equiv$ is a congruence with respect to the KAT operators and satisfies all the axioms of KAT except $p \cdot 0=0$.

Proof. All cases are straightforward except perhaps the axiom $q+r p \leq r \Rightarrow q p^{*} \leq r$, which we argue explicitly. Assuming

$$
R_{\alpha}(q+r p) \subseteq R_{\alpha}(r)
$$

for all $\alpha$, we wish to show that

$$
R_{\alpha}\left(q p^{*}\right) \subseteq R_{\alpha}(r)
$$

for all $\alpha$. This is true for $\alpha=0$ because the interpretation $R_{0}$ is a homomorphism. For $\alpha \neq 0$,

$$
\begin{aligned}
R_{\alpha}\left(q p^{*}\right) & =R_{\alpha}(q)+R_{0}(q) R_{0}(p)^{*} R_{\alpha}(p) \\
& =R_{\alpha}(q)+R_{0}\left(q p^{*}\right) R_{\alpha}(p) \\
& \subseteq R_{\alpha}(q)+R_{0}(r) R_{\alpha}(p) \\
& \subseteq R_{\alpha}(q)+R_{\alpha}(r)+R_{0}(r) R_{\alpha}(p) \\
& =R_{\alpha}(q+r p) \\
& \subseteq R_{\alpha}(r),
\end{aligned}
$$


the inclusion (3) from (2) for $\alpha=0$ and the inclusion (4) from (1).

The axiom $p \cdot 0=0$ is not satisfied, because $R_{1}(0)=0$ but $R_{1}$ (break $\left.\cdot 0\right)=1$.

Example 3.2 Let us show that

while $b$ do $p \equiv$ loop if $b$ then $p$ else break

provided $R_{n}(p)=0$ for all $n \geq 1$. Note that this is false without the proviso: for $p=$ break, the left-hand side is equivalent to if $b$ then break and the right-hand side is equivalent to skip. Thus break cannot be used to break out of a while loop.

The left-hand side reduces to $(b p)^{*} \bar{b}$, and it is not hard to show that

$$
R_{\alpha}\left((b p)^{*} \bar{b}\right)= \begin{cases}R_{0}(b p)^{*} R_{0}(\bar{b}), & \text { if } \alpha=0 \\ R_{0}(b p)^{*} R_{0}(b) R_{\alpha}(p), & \text { if } \alpha \neq 0 .\end{cases}
$$

For the right-hand side,

$$
\begin{aligned}
& R_{\alpha}(\text { loop if } b \text { then } p \text { else break }) \\
&=\quad R_{s(\alpha)}\left((\text { if } b \text { then } p \text { else break })^{*}\right) \\
&= R_{s(\alpha)}\left((b p+\bar{b} \text { break })^{*}\right) \\
&= R_{0}(b p+\bar{b} \text { break })^{*} R_{s(\alpha)}(b p+\bar{b} \text { break }) \\
&= R_{0}(b p)^{*} R_{0}(b) R_{s(\alpha)}(p) \\
&+R_{0}(b p)^{*} R_{0}(\bar{b}) R_{s(\alpha)}(\text { break }) \\
&=\left\{\begin{array}{cc}
R_{0}(b p)^{*} R_{0}(b) R_{1}(p) \\
+R_{0}(b p)^{*} R_{0}(\bar{b}) R_{1}(\text { break }), & \text { if } \alpha=0, \\
R_{0}(b p)^{*} R_{0}(b) R_{s(\alpha)}(p), & \text { if } \alpha \neq 0,
\end{array}\right. \\
&= \begin{cases}R_{0}(b p)^{*} R_{0}(\bar{b}), & \text { if } \alpha=0, \\
R_{0}(b p)^{*} R_{0}(b) R_{\alpha}(p), & \text { if } \alpha \neq 0,\end{cases}
\end{aligned}
$$

since $R_{1}(p)=0$ and $R_{s(\alpha)}(p)=R_{\alpha}(p)$ for $\alpha \neq 0$ under the assumption.

For the second part of the construction, let $\alpha$ be a continuation label and $\ell$ a statement label. Define the expressions $R_{\ell \alpha}(p)$ inductively as follows. Intuitively, $R_{\ell \alpha}(p)$ represents the computations of $p$ starting at the label $\ell$ and leading to the continuation $\alpha$.

$$
\begin{array}{rcl}
R_{\ell \alpha}(\ell: p) & \stackrel{\text { def }}{=} R_{\alpha}(p)+R_{\ell \alpha}(p) \\
R_{\ell \alpha}\left(\ell^{\prime}: p\right) & \stackrel{\text { def }}{=} & R_{\ell \alpha}(p), \ell^{\prime} \neq \ell \\
R_{\ell \alpha}(p+q) & \stackrel{\text { def }}{=} & R_{\ell \alpha}(p)+R_{\ell \alpha}(q) \\
R_{\ell \alpha}(p q) & \stackrel{\text { def }}{=} & R_{\ell \alpha}(p)+R_{\ell 0}(p) \cdot R_{\alpha}(q)+R_{\ell \alpha}(q) \\
R_{\ell \alpha}\left(p^{*}\right) & \stackrel{\text { def }}{=} & R_{\ell \alpha}(p)+R_{\ell 0}(p) \cdot R_{0}(p)^{*} \cdot R_{\alpha}(p) \\
R_{\ell \alpha}(p) & \stackrel{\text { def }}{=} & 0, p \text { a test, primitive action, } \\
& & \text { goto } \ell^{\prime}, \text { or break } n .
\end{array}
$$

For loop, we define

$$
\begin{aligned}
& R_{\ell \alpha}(\text { loop } p) \\
& \quad \stackrel{\text { def }}{=} R_{\ell, s(\alpha)}\left(p^{*}\right) \\
& \quad=R_{\ell, s(\alpha)}(p)+R_{\ell 0}(p) \cdot R_{0}(p)^{*} \cdot R_{s(\alpha)}(p) \\
& \quad=\quad R_{\ell, s(\alpha)}(p)+R_{\ell 0}(p) \cdot R_{\alpha}(\text { loop } p) .
\end{aligned}
$$

The statement labels $\ell$ need not have a unique occurrence in the program. If $\ell$ should have more than one occurrence, the interpretation is the natural nondeterministic one.

The KAT expressions $R_{\alpha \beta}(p)$ can be assembled into a square matrix $R(p)$ indexed by the continuation labels appearing in $p$; thus $R(p)_{\alpha \beta}=R_{\alpha \beta}(p)$. In addition, we include an extra row and column with index $s$ such that $R(p)_{s \alpha}=R_{\alpha}(p)$. Matrix entries not otherwise specified (e.g., $R(p)_{1,0}$ or $R(p)_{\ell s}$ ) are 0 . One can form $R(p)^{*}$ by the usual construction of the asterate of a square matrix over a KAT. The entry $R(p)_{s 0}^{*}$ is a KAT expression denoting the set of all halting computations of the program. This is the least fixpoint of a system of linear inequalities involving the $R_{\alpha \beta}(p)$.

\section{Basic Identities}

In this section we develop some basic consequences of the definitions of Section 3. These will be used in the results of Sections 5 and 6 .

First we prove some identities involving de Bruijn indices. Let $I_{m}$ be inductively defined functions on programs that behave as homomorphisms with respect to the KAT operators and are defined on the other constructs as follows:

$$
\begin{aligned}
I_{m}(\text { goto } \ell) & =\text { goto } \ell \\
I_{m}(\text { break } n) & = \begin{cases}\text { break } n, & \text { if } n<m, \\
\text { break } n+1, & \text { if } n \geq m\end{cases} \\
I_{m}(\text { loop } p) & =\text { loop }\left(I_{m+1}(p)\right) .
\end{aligned}
$$

Note that the $I_{m}$ are identity homomorphisms on pure KAT expressions.

The function $I_{1}$ is the one we are interested in, and the other $I_{m}$ are defined for auxiliary purposes. Intuitively, $I_{1}$ says to increment all the free de Bruijn indices by 1 . 
For example,

$$
\begin{aligned}
& I_{1}(\text { loop }(\text { loop }(\text { loop }(\text { break } 3)))) \\
& \quad=\text { loop }\left(\text { loop }\left(\text { loop }\left(I_{4}(\text { break } 3)\right)\right)\right) \\
& \quad=\text { loop }(\text { loop }(\text { loop }(\text { break } 3))) \\
& I_{1}(\text { loop }(\text { loop }(\text { break } 3))) \\
& \quad=\text { loop }\left(\text { loop }\left(I_{3}(\text { break } 3)\right)\right) \\
& \quad=\text { loop }(\text { loop }(\text { break } 4))
\end{aligned}
$$

In the first expression, the break 3 is bound, so it is not incremented, but in the second, it is free, so it is incremented. In general, an occurrence of a de Bruijn index $n$ is bound if it occurs in the scope of at least $n$ nested loop statements, otherwise it is free.

Lemma 4.1 For all $m \geq 1$ and $n \geq 0$,

$$
\begin{aligned}
R_{n}\left(I_{m}(p)\right) & = \begin{cases}R_{n}(p), & \text { if } n<m, \\
0, & \text { if } n=m, \\
R_{n-1}(p), & \text { if } n>m,\end{cases} \\
R_{\ell}\left(I_{m}(p)\right) & =R_{\ell}(p) .
\end{aligned}
$$

Proof. We prove this by induction on the structure of $p$. For the case $n=0$, we only need to show

$$
R_{0}\left(I_{m}(p)\right)=R_{0}(p) .
$$

For $p$ a test, atomic action, or goto $\ell, I_{m}(p)=p$, therefore (5) holds. For break $k$,

$$
\begin{aligned}
R_{0}\left(I_{m}(\text { break } k)\right) & = \begin{cases}R_{0}(\text { break } k), & \text { if } k<m, \\
R_{0}(\text { break } k+1), & \text { if } k \geq m\end{cases} \\
& =0 \\
& =R_{0}(\text { break } k) .
\end{aligned}
$$

For expressions formed from the operators,$+ \cdot$, and *, (5) follows from the induction hypothesis on smaller expressions and the fact that both $I_{m}$ and $R_{0}$ are homomorphisms with respect to these operators. Finally, for loop $p$,

$$
\begin{aligned}
R_{0}\left(I_{m}(\text { loop } p)\right) & =R_{0}\left(\text { loop } I_{m+1}(p)\right) \\
& =R_{1}\left(I_{m+1}(p)^{*}\right) \\
& =R_{1}\left(I_{m+1}\left(p^{*}\right)\right) \\
& =R_{1}\left(p^{*}\right) \\
& =R_{0}(\text { loop } p) .
\end{aligned}
$$

Now assume $n \geq 1$. As before, $R_{n}\left(I_{m}(p)\right)=R_{n}(p)$ for $p$ a test, atomic action, or goto $\ell$. For break $k$,

$$
\begin{aligned}
& R_{n}\left(I_{m}(\text { break } k)\right) \\
& = \begin{cases}R_{n}(\text { break } k), & \text { if } k<m, \\
R_{n}(\text { break } k+1), & \text { if } k \geq m\end{cases} \\
& = \begin{cases}1, \quad \text { if } n=k<m \text { or } n=k+1 \geq m+1, \\
0, \quad \text { otherwise }\end{cases} \\
& = \begin{cases}R_{n}(\text { break } k), & \text { if } n<m, \\
0, & \text { if } n=m, \\
R_{n-1}(\text { break } k), & \text { if } n>m .\end{cases}
\end{aligned}
$$

The case for the operator + follows from the linearity of $I_{m}$ and $R_{n}$. For $\cdot{ }^{*}$, and loop,

$$
\begin{aligned}
& R_{n}\left(I_{m}(p q)\right) \\
& =R_{n}\left(I_{m}(p) \cdot I_{m}(q)\right) \\
& =R_{n}\left(I_{m}(p)\right)+R_{0}\left(I_{m}(p)\right) \cdot R_{n}\left(I_{m}(q)\right) \\
& = \begin{cases}R_{n}(p)+R_{0}(p) \cdot R_{n}(q), & \text { if } n<m, \\
0+R_{0}(p) \cdot 0, & \text { if } n=m, \\
R_{n-1}(p)+R_{0}(p) \cdot R_{n-1}(q), & \text { if } n>m\end{cases} \\
& = \begin{cases}R_{n}(p q), & \text { if } n<m, \\
0, & \text { if } n=m, \\
R_{n-1}(p q), & \text { if } n>m,\end{cases} \\
& R_{n}\left(I_{m}\left(p^{*}\right)\right)=R_{n}\left(I_{m}(p)^{*}\right) \\
& =R_{0}\left(I_{m}(p)\right)^{*} \cdot R_{n}\left(I_{m}(p)\right) \\
& = \begin{cases}R_{0}(p)^{*} \cdot R_{n}(p), & \text { if } n<m, \\
R_{0}(p)^{*} \cdot 0, & \text { if } n=m, \\
R_{0}(p)^{*} \cdot R_{n-1}(p), & \text { if } n>m\end{cases} \\
& = \begin{cases}R_{n}\left(p^{*}\right), & \text { if } n<m, \\
0, & \text { if } n=m, \\
R_{n-1}\left(p^{*}\right), & \text { if } n>m,\end{cases} \\
& \begin{aligned}
R_{n}\left(I_{m}(\text { loop } p)\right) & =R_{n}\left(\text { loop } I_{m+1}(p)\right) \\
& =R_{n+1}\left(I_{m+1}(p)^{*}\right) \\
& =R_{n+1}\left(I_{m+1}\left(p^{*}\right)\right) \\
& = \begin{cases}R_{n+1}\left(p^{*}\right), & \text { if } n<m, \\
0, & \text { if } n=m, \\
R_{n}\left(p^{*}\right), & \text { if } n>m\end{cases} \\
& = \begin{cases}R_{n}(\text { loop } p), & \text { if } n<m, \\
0, & \text { if } n=m, \\
R_{n-1}(\text { loop } p) & \text { if } n>m .\end{cases}
\end{aligned}
\end{aligned}
$$

It remains to show that $R_{\ell}\left(I_{m}(p)\right)=R_{\ell}(p)$. This holds for tests, atomic actions, and goto instructions 
as above. For break $k$,

$$
\begin{aligned}
R_{\ell}\left(I_{m}(\text { break } k)\right) & = \begin{cases}R_{\ell}(\text { break } k), & \text { if } k<m, \\
R_{\ell}(\operatorname{break} k+1), & \text { if } k \geq m\end{cases} \\
& =0 \\
& =R_{\ell}(\operatorname{break} k) .
\end{aligned}
$$

The case for the operator + follows from the linearity of $I_{m}$ and $R_{\ell}$. For $\cdot{ }^{*}$, and loop,

$$
\begin{aligned}
R_{\ell}\left(I_{m}(p q)\right)= & R_{\ell}\left(I_{m}(p) \cdot I_{m}(q)\right) \\
= & R_{\ell}\left(I_{m}(p)\right)+R_{0}\left(I_{m}(p)\right) \cdot R_{\ell}\left(I_{m}(q)\right) \\
= & R_{\ell}(p)+R_{0}(p) \cdot R_{\ell}(q) \\
= & R_{\ell}(p q) \\
R_{\ell}\left(I_{m}\left(p^{*}\right)\right)= & R_{\ell}\left(I_{m}(p)^{*}\right) \\
= & R_{0}\left(I_{m}(p)\right)^{*} \cdot R_{\ell}\left(I_{m}(p)\right) \\
= & R_{0}(p)^{*} \cdot R_{\ell}(p) \\
= & R_{\ell}\left(p^{*}\right) \\
& =R_{\ell}\left(100 \mathrm{p} I_{m+1}(p)\right) \\
& =R_{\ell}\left(I_{m+1}(p)^{*}\right) \\
& =R_{\ell}\left(I_{m+1}\left(p^{*}\right)\right) \\
& =R_{\ell}\left(p^{*}\right) \\
& =R_{\ell}(\text { loop } p) .
\end{aligned}
$$

Corollary 4.2 For all $m \geq 1$ and $n \geq 0$,

$$
R_{\beta}(\text { loop } p)=R_{s(\beta)}\left(\text { loop }\left(I_{1}(p)\right)\right) .
$$

Proof. By the lemma,

$$
R_{s^{2}(\beta)}\left(I_{1}(p)\right)=R_{s(\beta)}(p) \quad R_{0}\left(I_{1}(p)\right)=R_{0}(p),
$$

thus

$$
\begin{aligned}
R_{\beta}(\text { loop } p) & =R_{s(\beta)}\left(p^{*}\right) \\
& =R_{0}(p)^{*} \cdot R_{s(\beta)}(p) \\
& =R_{0}\left(I_{1}(p)\right)^{*} \cdot R_{s^{2}(\beta)}\left(I_{1}(p)\right) \\
& =R_{s^{2}(\beta)}\left(I_{1}(p)^{*}\right) \\
& =R_{s(\beta)}\left(\text { loop }\left(I_{1}(p)\right)\right)
\end{aligned}
$$

Define $J_{m}=I_{m} \circ I_{m-1} \circ I_{m-2} \circ \cdots \circ I_{1}$.

Lemma 4.3 For all $m \geq 1$ and $n \geq 0$,

$$
\begin{aligned}
R_{n}\left(J_{m}(p) \cdot \operatorname{break} m\right) & = \begin{cases}R_{n-m}(p), & \text { if } n \geq m, \\
0, & \text { if } n<m\end{cases} \\
R_{\ell}\left(J_{m}(p) \cdot \operatorname{break} m\right) & =R_{\ell}(p) .
\end{aligned}
$$

In particular,

$$
R_{s(\alpha)}\left(I_{1}(p) \cdot \text { break }\right)=R_{\alpha}(p) .
$$

Proof. From Lemma 4.1 it follows inductively that for all $m \geq 1$ and $n \geq 0$,

$$
\begin{aligned}
R_{n}\left(J_{m}(p)\right) & = \begin{cases}R_{0}(p), & \text { if } n=0, \\
0, & \text { if } 0<n \leq m, \\
R_{n-m}(p), & \text { if } n>m,\end{cases} \\
R_{\ell}\left(J_{m}(p)\right) & =R_{\ell}(p) .
\end{aligned}
$$

For $n=0$,

$$
\begin{aligned}
R_{0}\left(J_{m}(p) \cdot \text { break } m\right) & =R_{0}\left(J_{m}(p)\right) \cdot R_{0}(\text { break } m) \\
& =R_{0}(p) \cdot 0 \\
& =0 .
\end{aligned}
$$

For $n>0$,

$$
\begin{aligned}
& R_{n}\left(J_{m}(p) \cdot \text { break } m\right) \\
& =R_{n}\left(J_{m}(p)\right)+R_{0}\left(J_{m}(p)\right) \cdot R_{n}(\text { break } m) \\
& =R_{n}\left(J_{m}(p)\right)+R_{0}(p) \cdot R_{n}(\text { break } m) \\
& = \begin{cases}0+R_{0}(p) \cdot 0, & \text { if } 1 \leq n<m, \\
0+R_{0}(p) \cdot 1, & \text { if } n=m, \\
R_{n-m}(p)+R_{0}(p) \cdot 0, & \text { if } n>m\end{cases} \\
& = \begin{cases}0, & \text { if } 1 \leq n<m, \\
R_{n-m}(p), & \text { if } n \geq m .\end{cases}
\end{aligned}
$$

For $\ell$,

$$
\begin{aligned}
& R_{\ell}\left(J_{m}(p) \cdot \text { break } m\right) \\
& \quad=R_{\ell}\left(J_{m}(p)\right)+R_{0}\left(J_{m}(p)\right) \cdot R_{\ell}(\text { break } m) \\
& \quad=R_{\ell}(p)+R_{0}(p) \cdot 0 \\
& \quad=R_{\ell}(p) .
\end{aligned}
$$

Lemma 4.4 loop $\left(I_{1}(p) \cdot\right.$ break $) \equiv p$.

Proof. For any $\alpha$,

$$
\begin{aligned}
& R_{\alpha}\left(\text { loop }\left(I_{1}(p) \cdot \text { break }\right)\right) \\
& \quad=R_{s(\alpha)}\left(\left(I_{1}(p) \cdot \text { break }\right)^{*}\right) \\
& =R_{s(\alpha)}\left(1+I_{1}(p) \cdot \text { break }\right) \\
& =R_{s(\alpha)}(1)+R_{s(\alpha)}\left(I_{1}(p) \cdot \text { break }\right) \\
& =R_{\alpha}(p) .
\end{aligned}
$$

Lemma 4.5 For any $q$,

$$
\begin{gathered}
R_{n}\left(\text { loop }\left(J_{2}(p) \cdot \text { break } 2\right) \cdot q\right) \\
\quad= \begin{cases}0, & \text { if } n=0, \\
R_{n-1}(p), & \text { if } n>0\end{cases}
\end{gathered}
$$




$$
R_{\ell}\left(\text { loop }\left(J_{2}(p) \cdot \text { break } 2\right) \cdot q\right)=R_{\ell}(p) .
$$

In particular,

$$
R_{s(\alpha)}\left(\text { loop }\left(J_{2}(p) \cdot \text { break } 2\right) \cdot q\right)=R_{\alpha}(p) .
$$

Proof.

$$
\begin{aligned}
& R_{0}\left(\text { loop }\left(J_{2}(p) \cdot \text { break } 2\right) \cdot q\right) \\
& \quad=R_{0}\left(\text { loop }\left(J_{2}(p) \cdot \text { break } 2\right)\right) \cdot R_{0}(q) \\
& =R_{0}\left(J_{2}(p) \cdot \text { break } 2\right)^{*} \cdot R_{1}\left(J_{2}(p) \cdot \text { break } 2\right) \cdot R_{0}(q) \\
& =0^{*} \cdot 0 \cdot R_{0}(q) \\
& =0,
\end{aligned}
$$

and for any $\alpha$,

$$
\begin{aligned}
R_{s(\alpha)} & \text { loop } \left.\left(J_{2}(p) \cdot \text { break } 2\right) \cdot q\right) \\
= & R_{s(\alpha)}\left(\text { loop }\left(J_{2}(p) \cdot \text { break } 2\right)\right) \\
& +R_{0}\left(\text { loop }\left(J_{2}(p) \cdot \text { break } 2\right)\right) \cdot R_{n}(q) \\
= & R_{s(s(\alpha))}\left(\left(J_{2}(p) \cdot \text { break } 2\right)^{*}\right) \\
& +R_{1}\left(\left(J_{2}(p) \cdot \text { break } 2\right)^{*}\right) \cdot R_{n}(q) \\
= & R_{s(s(\alpha))}\left(1+J_{2}(p) \cdot \text { break } 2\right) \\
& +R_{1}\left(1+J_{2}(p) \cdot \text { break } 2\right) \cdot R_{n}(q) \\
= & R_{s(s(\alpha))}\left(J_{2}(p) \cdot \text { break } 2\right)+0 \cdot R_{n}(q) \\
= & R_{s(s(\alpha))}\left(J_{2}(p) \cdot \text { break } 2\right) \\
= & R_{\alpha}(p) .
\end{aligned}
$$

Lemma 4.6 For any $q$,

$$
\text { loop }\left(\text { loop }\left(J_{2}(p) \cdot \operatorname{break} 2\right) \cdot q\right) \equiv p .
$$

Proof.

$$
\begin{aligned}
& R_{\alpha}\left(\text { loop }\left(\text { loop }\left(J_{2}(p) \cdot \text { break } 2\right) \cdot q\right)\right) \\
&= R_{0}\left(\text { loop }\left(J_{2}(p) \cdot \text { break } 2\right) \cdot q\right)^{*} \\
& \cdot R_{s(\alpha)}\left(\text { loop }\left(J_{2}(p) \cdot \text { break } 2\right) \cdot q\right) \\
&= 0^{*} \cdot R_{\alpha}(p) \\
&= R_{\alpha}(p) .
\end{aligned}
$$

Let $K_{\ell, m}, m \geq 1$, be a family of substitution operators that act as the identity on all tests and atomic actions except goto $\ell$, and act as a KAT homomorphism on all operators except loop. For the remaining two constructs,

$$
\begin{aligned}
K_{\ell, m}(\text { goto } \ell) & =\text { break } m \\
K_{\ell, m}(\text { loop } p) & =\text { loop }\left(K_{\ell, m+1}(p)\right)
\end{aligned}
$$

That is, $K_{\ell, m}$ substitutes break $m+k$ for each occurrence of goto $\ell$ of loop-depth $k$.

\section{Lemma 4.7}

loop (loop $\left(K_{\ell, 1}\left(J_{2}(p)\right) \cdot\right.$ break 2$) \cdot$ goto $\left.\ell\right) \equiv p$.

Proof. By Lemma 4.6, it suffices to show

$$
\begin{aligned}
& \text { loop (loop } \left.\left(K_{\ell, 1}\left(J_{2}(p)\right) \cdot \text { break } 2\right) \cdot \text { goto } \ell\right) \\
& \equiv \text { loop }\left(\text { loop }\left(J_{2}(p) \cdot \text { break } 2\right)\right) \text {. }
\end{aligned}
$$

Consequently, it suffices to show

$$
\begin{aligned}
& \text { loop }\left(K_{\ell, 1}\left(J_{2}(p)\right) \cdot \text { break } 2\right) \cdot \text { goto } \ell \\
& \equiv \text { loop }\left(J_{2}(p) \cdot \text { break } 2\right) .
\end{aligned}
$$

We need to show

$$
\begin{aligned}
& R_{\alpha}\left(\text { loop }\left(K_{\ell, 1}\left(J_{2}(p)\right) \cdot \text { break } 2\right) \cdot \text { goto } \ell\right) \\
& \quad=R_{\alpha}\left(\text { loop }\left(J_{2}(p) \cdot \text { break } 2\right)\right) .
\end{aligned}
$$

We have already shown in Lemma 4.5 that

$$
\begin{aligned}
R_{0}\left(\text { loop }\left(J_{2}(p) \cdot \text { break } 2\right)\right) & =0 \\
R_{s(\alpha)}\left(\text { loop }\left(J_{2}(p) \cdot \text { break } 2\right)\right) & =R_{\alpha}(p) .
\end{aligned}
$$

For $n=0$,

$$
\begin{aligned}
& R_{0}\left(\text { loop }\left(K_{\ell, 1}\left(J_{2}(p)\right) \cdot \text { break } 2\right) \cdot \text { goto } \ell\right) \\
& \quad=R_{0}\left(\text { loop }\left(K_{\ell, 1}\left(J_{2}(p)\right) \cdot \text { break } 2\right)\right) \cdot R_{0}(\text { goto } \ell) \\
& =R_{0}\left(\text { loop }\left(K_{\ell, 1}\left(J_{2}(p)\right) \cdot \text { break } 2\right)\right) \cdot 0 \\
& =0 .
\end{aligned}
$$

For $\alpha \neq \ell$,

$$
\begin{aligned}
R_{s(\alpha)} & \text { loop } \left.\left(K_{\ell, 1}\left(J_{2}(p)\right) \cdot \text { break } 2\right) \cdot \text { goto } \ell\right) \\
= & R_{s(\alpha)}\left(\text { loop }\left(K_{\ell, 1}\left(J_{2}(p)\right) \cdot \text { break } 2\right)\right) \\
& +R_{0}\left(\text { loop }\left(K_{\ell, 1}\left(J_{2}(p)\right) \cdot \text { break } 2\right)\right) \cdot R_{s(\alpha)}(\text { goto } \ell) \\
= & R_{s(s(\alpha))}\left(K_{\ell, 1}\left(J_{2}(p)\right) \cdot \text { break } 2\right) \\
& +R_{1}\left(K_{\ell, 1}\left(J_{2}(p)\right) \cdot \text { break } 2\right) \cdot 0 \\
= & R_{s(s(\alpha))}\left(K_{\ell, 1}\left(J_{2}(p) \cdot \text { break } 2\right)\right) \\
= & R_{s(s(\alpha))}\left(J_{2}(p) \cdot \text { break } 2\right) \\
= & R_{\alpha}(p) .
\end{aligned}
$$

Finally, for $\ell$,

$$
\begin{aligned}
& R_{\ell}\left(\text { loop }\left(K_{\ell, 1}\left(J_{2}(p)\right) \cdot \text { break } 2\right) \cdot \text { goto } \ell\right) \\
&= R_{\ell}\left(\text { loop }\left(K_{\ell, 1}\left(J_{2}(p)\right) \cdot \text { break } 2\right)\right) \\
&+R_{0}\left(\text { loop }\left(K_{\ell, 1}\left(J_{2}(p)\right) \cdot \text { break } 2\right)\right) \cdot R_{\ell}(\text { goto } \ell) \\
&= R_{\ell}\left(K_{\ell, 1}\left(J_{2}(p)\right) \cdot \text { break } 2\right) \\
&+R_{1}\left(K_{\ell, 1}\left(J_{2}(p)\right) \cdot \text { break } 2\right) \cdot 1 \\
&= R_{\ell}\left(K_{\ell, 1}\left(J_{2}(p) \cdot \text { break } 2\right)\right) \\
&+R_{1}\left(K_{\ell, 1}\left(J_{2}(p) \cdot \text { break } 2\right)\right) \\
&= 0+R_{1}\left(J_{2}(p) \cdot \text { break } 2\right)+R_{\ell}\left(J_{2}(p) \cdot \text { break } 2\right) \\
&= R_{\ell}\left(J_{2}(p) \cdot \text { break } 2\right) \\
&= R_{\ell}(p) .
\end{aligned}
$$




\subsection{Lifting Labels}

In general, programs might have labels embedded deeply in them. In this section we perform some transformations to move labels to the outermost level. We show that for each label $\ell$ and program $p$, there is a program $p_{\ell}$ such that for all $\beta, R_{\ell \beta}(p)=R_{\beta}\left(p_{\ell}\right)$. Intuitively, jumping into the middle of $p$ at label $\ell$ is the same as running $p_{\ell}$ from the beginning. Moreover, if $p$ is deterministic, then so is $p_{\ell}$.

Let $p$ be a program and $\ell$ a label. Define

$$
\begin{aligned}
& p_{\ell} \stackrel{\text { def }}{=} 0 \text {, for } p \text { a test, atomic program, } \\
& \text { break } n \text {, or goto } \ell^{\prime} \text {, } \\
& (p+q)_{\ell} \stackrel{\text { def }}{=} p_{\ell}+q_{\ell}, \\
& (p q)_{\ell} \stackrel{\text { def }}{=} p_{\ell} q+q_{\ell} \\
& \left(p^{*}\right)_{\ell} \stackrel{\text { def }}{=} p_{\ell} p^{*}, \\
& \left(\ell^{\prime}: p\right)_{\ell} \stackrel{\text { def }}{=} \begin{cases}p+p_{\ell}, & \text { if } \ell=\ell^{\prime}, \\
p_{\ell}, & \text { if } \ell \neq \ell^{\prime},\end{cases} \\
& (\text { loop } p)_{\ell} \stackrel{\text { def }}{=} \text { loop }\left(p_{\ell} \cdot \text { loop }\left(I_{1}(p)\right)\right) \text {. }
\end{aligned}
$$

Theorem 4.8 For all $\ell, \beta$, and $p$,

$$
R_{\ell \beta}(p)=R_{\beta}\left(p_{\ell}\right) .
$$

Proof. The arguments for tests, atomic programs, break $n$, goto $\ell^{\prime}, p+q$, and $\ell^{\prime}: p$ are straightforward.

For $p q$ and $p^{*}$,

$$
\begin{aligned}
& R_{\ell \beta}(p q) \\
& = \begin{cases}R_{\ell \beta}(p)+R_{\ell 0}(p) \cdot R_{\beta}(q)+R_{\ell \beta}(q), & \text { if } \beta \neq 0, \\
R_{\ell 0}(p) \cdot R_{0}(q)+R_{\ell 0}(q), & \text { if } \beta=0\end{cases} \\
& = \begin{cases}R_{\beta}\left(p_{\ell}\right)+R_{0}\left(p_{\ell}\right) \cdot R_{\beta}(q)+R_{\beta}\left(q_{\ell}\right), & \text { if } \beta \neq 0, \\
R_{0}\left(p_{\ell}\right) \cdot R_{0}(q)+R_{0}\left(q_{\ell}\right), & \text { if } \beta=0\end{cases} \\
& = \begin{cases}R_{\beta}\left(p_{\ell} q\right)+R_{\beta}\left(q_{\ell}\right), & \text { if } \beta \neq 0, \\
R_{0}\left(p_{\ell} q\right)+R_{0}\left(q_{\ell}\right), & \text { if } \beta=0\end{cases} \\
& =R_{\beta}\left(p_{\ell} q+q_{\ell}\right) \\
& =R_{\beta}\left((p q)_{\ell}\right), \\
& R_{\ell \beta}\left(p^{*}\right) \\
& = \begin{cases}R_{\ell \beta}(p)+R_{\ell 0}(p) \cdot R_{0}(p)^{*} \cdot R_{\beta}(p), & \text { if } \beta \neq 0, \\
R_{\ell 0}(p) \cdot R_{0}(p)^{*}, & \text { if } \beta=0\end{cases} \\
& = \begin{cases}R_{\beta}\left(p_{\ell}\right)+R_{0}\left(p_{\ell}\right) \cdot R_{\beta}\left(p^{*}\right), & \text { if } \beta \neq 0, \\
R_{0}\left(p_{\ell}\right) \cdot R_{0}\left(p^{*}\right), & \text { if } \beta=0\end{cases} \\
& =R_{\beta}\left(p_{\ell} p^{*}\right) \\
& =R_{\beta}\left(\left(p^{*}\right)_{\ell}\right) .
\end{aligned}
$$

Finally, for loop $p$, first observe that if $R_{0}(q)=0$, then

$$
\begin{aligned}
R_{\beta}\left(q^{*}\right) & =R_{\beta}(1)+R_{0}(q)^{*} \cdot R_{\beta}(q) \\
& =R_{\beta}(1)+R_{\beta}(q) \\
& = \begin{cases}1, & \text { if } \beta=0, \\
R_{\beta}(q), & \text { if } \beta \neq 0 .\end{cases}
\end{aligned}
$$

We will apply this with $q=p_{\ell} \cdot$ loop $\left(I_{1}(p)\right)$, observing that

$$
\begin{aligned}
R_{0}\left(\text { loop }\left(I_{1}(p)\right)\right) & =R_{1}\left(I_{1}(p)^{*}\right) \\
& =R_{0}\left(I_{1}(p)\right)^{*} \cdot R_{1}\left(I_{1}(p)\right)=0,
\end{aligned}
$$

hence

$$
\begin{aligned}
& \quad R_{0}\left(p_{\ell} \cdot \text { loop }\left(I_{1}(p)\right)\right) \\
& \quad=R_{0}\left(p_{\ell}\right) \cdot R_{0}\left(\text { loop }\left(I_{1}(p)\right)\right)=0 . \\
& R_{\ell \beta}(\text { loop } p) \\
& =R_{\ell, s(\beta)}\left(p^{*}\right) \\
& =R_{\ell, s(\beta)}(p)+R_{\ell 0}(p) \cdot R_{0}(p)^{*} \cdot R_{s(\beta)}(p) \\
& =R_{s(\beta)}\left(p_{\ell}\right)+R_{0}\left(p_{\ell}\right) \cdot R_{s(\beta)}\left(p^{*}\right) \\
& =R_{s(\beta)}\left(p_{\ell}\right)+R_{0}\left(p_{\ell}\right) \cdot R_{\beta}(\text { loop } p) \\
& =R_{s(\beta)}\left(p_{\ell}\right)+R_{0}\left(p_{\ell}\right) \cdot R_{s(\beta)}\left(\text { loop }\left(I_{1}(p)\right)\right) \\
& =R_{s(\beta)}\left(p_{\ell} \cdot \text { loop }\left(I_{1}(p)\right)\right) \\
& =R_{s(\beta)}\left(\left(p_{\ell} \cdot \text { loop }\left(I_{1}(p)\right)\right)^{*}\right) \\
& =R_{\beta}\left(\text { loop }\left(p_{\ell} \cdot \text { loop }\left(I_{1}(p)\right)\right)\right) .
\end{aligned}
$$

Lemma 4.9 If p contains no occurrence of $\ell$ as a label, that is, if $p$ contains no subprogram of the form $\ell: q$, then $p_{\ell} \equiv 0$.

Proof. This can be shown by induction on the structure of the program. All cases are quite obvious except perhaps for the case loop $p$. For this case, it suffices to show that if $p_{\ell} \equiv 0$, then (loop $\left.p\right)_{\ell} \equiv 0$.

$$
\begin{aligned}
(\text { loop } p)_{\ell} & =\text { loop }\left(p_{\ell} \cdot \text { loop }\left(I_{1}(p)\right)\right) \\
& \equiv \text { loop }\left(0 \cdot \text { loop }\left(I_{1}(p)\right)\right) \\
& \equiv \text { loop } 0
\end{aligned}
$$

and for any $\beta$,

$$
R_{\beta}(\text { loop } 0)=R_{s(\beta)}\left(0^{*}\right)=R_{s(\beta)}(1)=0 .
$$

Note that $p_{\ell}$ contains no occurrence of $\ell$ as a label, since in no case in the inductive definition of $p_{\ell}$ does $\ell$ occur on the right-hand side; thus $\left(p_{\ell}\right)_{\ell} \equiv 0$. 
Lemma 4.10 If $p$ is deterministic, then so is $p_{\ell}$.

Proof. This is true for $p$ a test, atomic program, break $n$, or goto $\ell^{\prime}$, since $p_{\ell}=0$, since 0 is deterministic. The argument for loop $p$ is also clear by the form of the right-hand side and the induction hypothesis.

For the case while $b$ do $p$, using the encoding $(b p)^{*} \bar{b}$ and unwinding the definitions, one can show that

$$
(\text { while } b \text { do } p)_{\ell}=p_{\ell} \cdot \text { while } b \text { do } p \text {. }
$$

This is deterministic by the induction hypothesis.

For the case if $b$ then $p$ else $q$, using the encoding $b p+\bar{b} q$ and unwinding the definitions, one can show that

$$
\text { (if } b \text { then } p \text { else } q)_{\ell}=p_{\ell}+q_{\ell} \text {. }
$$

By determinacy, at most one of $p$ or $q$ contains $\ell$ as a label. By Lemma 4.9, at least one of $p_{\ell}$ or $q_{\ell}$ is equivalent to 0 , so the entire program is equivalent to the other. Since $p$ and $q$ are deterministic, both $p_{\ell}$ and $q_{\ell}$ are deterministic by the induction hypothesis.

For the case $p q$, we have $(p q)_{\ell}=p_{\ell} q+q_{\ell}$. By the same argument as the previous case, the entire program is equivalent to either $p_{\ell} q$ or $q_{\ell}$, both of which are deterministic by the induction hypothesis.

Finally, for $\ell: p$, we have

$$
(\ell: p)_{\ell}=p+p_{\ell} \equiv p
$$

since by determinacy, $p_{\ell}$ contains no occurrence of $\ell$ as a label, therefore $p_{\ell} \equiv 0$.

\subsection{Modules}

For convenience, we extend the language with the following construct. If $p_{1}, \ldots, p_{n}$ are programs, then so is $\left(p_{1}, \ldots, p_{n}\right)$. The first program $p_{1}$ in the sequence is the main program and the $p_{1}, \ldots, p_{n}$ are called modules. For all $\alpha$ and $\ell$, define

$$
\begin{array}{rll}
R_{\alpha}\left(p_{1}, \ldots, p_{n}\right) & \stackrel{\text { def }}{=} & R_{\alpha}\left(p_{1}\right), \\
R_{\ell \alpha}\left(p_{1}, \ldots, p_{n}\right) & \stackrel{\text { def }}{=} & R_{\ell \alpha}\left(p_{1}\right)+\cdots+R_{\ell \alpha}\left(p_{n}\right) .
\end{array}
$$

Thus the entry point of $\left(p_{1}, \ldots, p_{n}\right)$ is the entry point of $p_{1}$, but other modules may be accessible by unconditional jumps goto $\ell$. If the program is deterministic, so that $\ell$ occurs in at most one module $p_{i}$, then $R_{\ell \alpha}\left(p_{1}, \ldots, p_{n}\right)=R_{\ell \alpha}\left(p_{i}\right)$. A program of the form $\left(p_{1}, \ldots, p_{n}\right)$ is called modular.

\section{Lemma 4.11}

$$
\begin{aligned}
& \left(p_{1}, \ldots, p_{n}\right) \\
& \quad \equiv \text { loop }\left(I_{1}\left(p_{1}\right) ; \text { break; } \cdots ; I_{1}\left(p_{n}\right) ; \text { break }\right) .
\end{aligned}
$$

This allows the construct $\left(p_{1}, \ldots, p_{n}\right)$ to be eliminated if desired. We omit the proof, since this fact is not needed in the remaining sections.

\section{Loops Programs are Sufficient}

In this section we use our calculus to give a formal equational proof that every propositional deterministic flowchart is equivalent to a loop program with multilevel breaks but without unconditional jumps. This is a folklore result that has been known since at least the early 1970s [14, 26], but to our knowledge has never been treated with this level of rigor. A somewhat different conversion was given in [25] in the same formalism, but without proof.

We must actually start not with a flowchart or automaton, but with an equivalent program expression, since our calculus works only with expressions, not with automata. However, there is a straightforward construction to convert a given strictly deterministic automaton as described in Section 2.4 to a modular program with unconditional jumps, which we sketch without proof.

Given a strictly deterministic automaton $M$, construct a modular program with one module for each state. The module corresponding to state $\ell$ has label $\ell$. The module corresponding to the start state occurs first in the modular program. For each state $\ell$, let $\alpha_{1}, \ldots, \alpha_{m}$ be all atoms such that $\delta(\ell)\left(\alpha_{i}\right)=\left(p_{i}, \ell_{i}\right)$ and let $\alpha_{m+1}, \ldots, \alpha_{n}$ be all atoms such that $\delta(\ell)\left(\alpha_{i}\right)=$ halt. The module corresponding to $\ell$ is

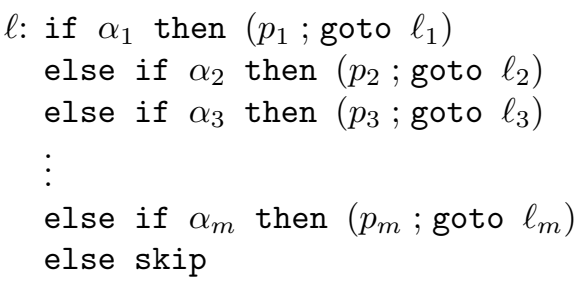

One can show without much difficulty that

$$
R_{\ell \beta}= \begin{cases}\alpha_{i} p_{i}, & \text { if } \beta=\ell_{i}, 1 \leq i \leq m, \\ \alpha_{m+1}+\cdots+\alpha_{n}, & \text { if } \beta=0, \\ 0, & \text { if } \beta>0 .\end{cases}
$$

It follows inductively that

$$
\mathrm{GS}\left(R_{\ell 0}^{*}\right)=\left\{\operatorname{gs}(\ell)(\sigma) \mid \sigma \in \mathrm{At}^{\omega}\right\} \cap(\mathrm{At} \cdot \Sigma)^{*} \cdot \mathrm{At},
$$


thus

$$
\begin{aligned}
\mathrm{GS}\left(R_{s 0}^{*}\right) & =\left\{\mathrm{gs}(\text { start })(\sigma) \mid \sigma \in \mathrm{At}^{\omega}\right\} \cap(\mathrm{At} \cdot \Sigma)^{*} \cdot \mathrm{At} \\
& =\mathrm{GS}(M) .
\end{aligned}
$$

Although our calculus cannot be used for this part of the construction, a formal semantic proof can be given along the lines sketched above.

Now assume we are given a deterministic program containing any of the programming constructs defined in Section 2.5, including while loops, unconditional jumps, loop instructions, or multilevel breaks.

Lemma 5.1 Consider two deterministic modular programs with $\ell$ th modules

$$
\ell \text { : loop }(q \text {; goto } \ell) \quad \ell \text { : loop } q \text {, }
$$

respectively, where $R_{\ell}(q)=0$. The two programs are otherwise identical. Let $F$ and $G$ be the matrices corresponding to these two programs, respectively, as described in Section 3. Let $\alpha, \beta$ be two continuation labels, $\beta \neq \ell$. Then $F_{\alpha \beta}^{*}=G_{\alpha \beta}^{*}$. In particular, $F_{s 0}^{*}=G_{s 0}^{*}$, therefore the two programs are equivalent.

Proof. Decompose the matrices $F$ and $G$ as

$$
F=\left[\begin{array}{cc}
F_{\ell \ell} & F^{\prime} \\
C & D
\end{array}\right] \quad G=\left[\begin{array}{cc}
G_{\ell \ell} & G^{\prime} \\
C & D
\end{array}\right]
$$

where $D$ is the square matrix obtained by deleting the th row and column of $F$ or $G(C$ and $D$ are the same in each case, by assumption). If $\alpha \neq \ell$, then

$$
\begin{aligned}
& F_{\alpha \beta}^{*}=\left(D+C F_{\ell \ell}^{*} F^{\prime}\right)_{\alpha \beta}^{*} \\
& G_{\alpha \beta}^{*}=\left(D+C G_{\ell \ell}^{*} G^{\prime}\right)_{\alpha \beta}^{*},
\end{aligned}
$$

and

$$
\begin{aligned}
& F_{\ell \beta}^{*}=\left(F_{\ell \ell}^{*} F^{\prime}\left(D+C F_{\ell \ell}^{*} F^{\prime}\right)^{*}\right)_{\ell \beta} \\
& G_{\ell \beta}^{*}=\left(G_{\ell \ell}^{*} G^{\prime}\left(D+C G_{\ell \ell}^{*} G^{\prime}\right)^{*}\right)_{\ell \beta} .
\end{aligned}
$$

In either case, it suffices to show

$$
F_{\ell \ell}^{*} F^{\prime}=G_{\ell \ell}^{*} G^{\prime},
$$

or in other words,

$$
F_{\ell \ell}^{*} F_{\ell \beta}=G_{\ell \ell}^{*} G_{\ell \beta}
$$

for all $\beta \neq \ell$. But

$$
\begin{aligned}
F_{\ell \ell} & =R_{\ell}(\text { loop }(q ; \text { goto } \ell))=R_{0}(q) \\
F_{\ell \beta} & =R_{\beta}(\text { loop }(q ; \text { goto } \ell))=R_{s(\beta)}(q) \\
G_{\ell \ell} & =R_{\ell}(\text { loop } q)=0 \\
G_{\ell \beta} & =R_{\beta}(\text { loop } q)=R_{0}(q)^{*} R_{s(\beta)}(q),
\end{aligned}
$$

so both sides of $(6)$ are $R_{0}(q)^{*} R_{s(\beta)}(q)$.
Lemma 5.2 Consider a deterministic modular program with distinct modules

$$
\text { loop }(q \text {; goto } \ell) \quad \ell \text { : loop }(r \text {; break })
$$

where $R_{\ell}(q)=R_{\ell}(r)=0$. Consider another program that is identical to the first, except with the left-hand module of (7) replaced by

$$
\text { loop }(q ; r ; \text { break }) \text {. }
$$

Let $F$ and $G$ be the matrices corresponding to these two programs, respectively. Let $\alpha, \beta$ be two continuation labels, $\beta \neq \ell$. Then $F_{\alpha \beta}^{*}=G_{\alpha \beta}^{*}$. In particular, $F_{s 0}^{*}=$ $G_{s 0}^{*}$, therefore the two programs are equivalent.

Proof. By a matrix decomposition argument similar to the one in the proof of Lemma 5.1, it suffices to show that

$$
F_{\alpha \beta}+F_{\alpha \ell} F_{\ell \beta}=G_{\alpha \beta} .
$$

By Theorem 4.8, we can assume without loss of generality that $\alpha$ is the label of the left-hand module of (7) in $F$ and the module (8) of $G$, which is either $s$ or an explicit label. Then

$$
\begin{aligned}
F_{\alpha \beta}= & R_{\beta}(\text { loop }(q ; \text { goto } \ell))=R_{s(\beta)}(q) \\
F_{\alpha \ell}= & R_{\ell}(\text { loop }(q ; \text { goto } \ell))=R_{0}(q) \\
F_{\ell \beta}= & R_{\beta}(\text { loop }(r ; \text { break })) \\
= & R_{s(\beta)}(r)+R_{0}(r) R_{s(\beta)}(\text { break }) \\
G_{\alpha \beta}= & R_{\beta}(\text { loop }(q ; r ; \text { break })) \\
= & R_{s(\beta)}(q)+R_{0}(q) R_{s(\beta)}(r) \\
& +R_{0}(q) R_{0}(r) R_{s(\beta)}(\text { break }),
\end{aligned}
$$

from which (9) follows.

Theorem 5.3 Every program is equivalent to a loop program with multilevel breaks but without unconditional jumps.

Proof. Starting from an arbitrary program $p$ with unconditional jumps, first use Theorem 4.8 to rewrite the program as a modular program $\left(p_{1}, \ldots, p_{n}\right)$, where $p_{1}$ is $p$ (without the initial label if it exists) and $p_{2}, \ldots, p_{n}$ are all programs of the form $\ell: p_{\ell}$ obtained from the lifting construction of Section 4.1 for all labels $\ell$ occurring in the program. By Theorem $4.8,\left(p_{1}, \ldots, p_{n}\right)$ and $p$ have the same matrix.

Now consider the last module in the list, and say it has label $\ell$. By Lemma 4.7, it can be written as

$$
\ell \text { : loop ( } s \text {; goto } \ell \text { ), }
$$


where $R_{\ell}(s)=0$. By Lemma 5.1 , this can be replaced by $\ell$ : loop $s$. Using Lemma 4.4 , this module can be rewritten as

$$
\ell \text { : loop }(r ; \text { break })
$$

where $R_{\ell}(r)=0$. Now for every other module besides this one, use Lemma 4.7 to rewrite it in the form

$$
\text { loop }(q ; \text { goto } \ell)
$$

where $R_{\ell}(q)=0$, then Lemma 5.2 to rewrite it in the form

$$
\text { loop }(q ; r ; \text { break })
$$

At this point there are no longer any occurrences of goto $\ell$ in the program, so the column of the matrix corresponding to $\ell$ is 0 . The row and column corresponding to $\ell$ can thus be deleted without changing the semantics of the program; the resulting matrix is that of the modular program with the last module deleted.

Continuing in this fashion, we can delete all modules except the first. We are left with a loop program with multilevel breaks and no unconditional jumps.

\section{Completeness}

The following theorem is a straightforward consequence of the completeness of KAT, but still bears mentioning:

Theorem 6.1 The calculus presented in this paper is sufficient to prove all valid identities between loop programs with multilevel breaks.

Proof. The inductive definitions of the loop and break $n$ constructs allow any program $p$ to be reduced to a matrix $R(p)$ of KAT expressions. The meaning of $p$ is defined to be the same as a particular entry of the asterate of that matrix, namely $R(p)_{s 0}^{*}$. Since KAT is complete for the equational theory of the guarded string model, it can prove the equivalence of two such translations if indeed the two programs represent the same set of guarded strings.

\section{Conclusion and Open Problems}

We have shown how to handle programming constructs involving nonlocal flow of control such as goto and multilevel break instructions in a simple propositional equational system. This fits well with the vision that simple equational reasoning suffices to handle a large class of basic program analysis tasks.

Some interesting open problems present themselves. Can one formulate a simple coalgebraic treatment of nonlocal flow of control involving a definition of the Brzozowski derivative [4] for the nonlocal control flow constructs? If so, it may be possible to given even simpler constructions and proofs. To what extent is it possible to extend the method to reason in the presence of commutativity conditions and other basic premises? And finally, it is clear that in principle, equivalence proofs be extracted automatically by reduction to KAT $[5,27]$, but can this process be streamlined by treating the nonlocal control flow constructs directly?

\section{Acknowledgements}

Thanks to Maksim Orlovich and Dustin Tseng for valuable conversations. This work was supported by NSF grant CCF-0635028.

\section{References}

[1] A. Angus and D. Kozen. Kleene algebra with tests and program schematology. Technical Report TR20011844, Computer Science Department, Cornell University, July 2001.

[2] E. Ashcroft and Z. Manna. The translation of goto programs into while programs. In C. Freiman, J. Griffith, and J. Rosenfeld, editors, Proceedings of IFIP Congress 71, volume 1, pages 250-255. North-Holland, 1972.

[3] A. Barth and D. Kozen. Equational verification of cache blocking in LU decomposition using Kleene algebra with tests. Technical Report TR2002-1865, Computer Science Department, Cornell University, June 2002.

[4] J. A. Brzozowski. Derivatives of regular expressions. J. Assoc. Comput. Mach., 11:481-494, 1964.

[5] H. Chen and R. Pucella. A coalgebraic approach to Kleene algebra with tests. Electronic Notes in Theoretical Computer Science, 82(1), 2003.

[6] E. Cohen. Hypotheses in Kleene algebra. Technical Report TM-ARH-023814, Bellcore, 1993. http://citeseer.nj.nec.com/1688.html.

[7] E. Cohen. Lazy caching in Kleene algebra, 1994. http://citeseer.nj.nec.com/22581.html.

[8] E. Cohen, D. Kozen, and F. Smith. The complexity of Kleene algebra with tests. Technical Report TR961598, Computer Science Department, Cornell University, July 1996. 
[9] J. H. Conway. Regular Algebra and Finite Machines. Chapman and Hall, London, 1971.

[10] N. G. de Bruijn. Lambda calculus notation with nameless dummies: A tool for automatic formula manipulation, with application to the Church-Rosser theorem. Indagationes Mathematicae, 34:381-392, 1972.

[11] K. Iwano and K. Steiglitz. A semiring on convex polygons and zero-sum cycle problems. SIAM J. Comput., 19(5):883-901, 1990.

[12] D. M. Kaplan. Regular expressions and the equivalence of programs. J. Comput. Syst. Sci., 3:361-386, 1969.

[13] S. C. Kleene. Representation of events in nerve nets and finite automata. In C. E. Shannon and J. McCarthy, editors, Automata Studies, pages 3-41. Princeton University Press, Princeton, N.J., 1956.

[14] S. R. Kosaraju. Analysis of structured programs. In Proc. 5th ACM Symp. Theory of Computing (STOC'73), pages 240-252, New York, NY, USA, 1973. ACM.

[15] Ł. Kot and D. Kozen. Second-order abstract interpretation via Kleene algebra. Technical Report TR20041971, Computer Science Department, Cornell University, December 2004.

[16] Ł. Kot and D. Kozen. Kleene algebra and bytecode verification. In F. Spoto, editor, Proc. 1st Workshop Bytecode Semantics, Verification, Analysis, and Transformation (Bytecode'05), pages 201-215, April 2005.

[17] D. Kozen. A completeness theorem for Kleene algebras and the algebra of regular events. Infor. and Comput., 110(2):366-390, May 1994.

[18] D. Kozen. Kleene algebra with tests. Transactions on Programming Languages and Systems, 19(3):427-443, May 1997.

[19] D. Kozen. On Hoare logic and Kleene algebra with tests. Trans. Computational Logic, 1(1):60-76, July 2000.

[20] D. Kozen. Automata on guarded strings and applications. Matématica Contemporânea, 24:117-139, 2003.

[21] D. Kozen. Kleene algebras with tests and the static analysis of programs. Technical Report TR2003-1915, Computer Science Department, Cornell University, November 2003.

[22] D. Kozen. On the coalgebraic theory of Kleene algebra with tests. Technical Report http://hdl. handle.net/1813/10173, Computing and Information Science, Cornell University, March 2008.

[23] D. Kozen and M.-C. Patron. Certification of compiler optimizations using Kleene algebra with tests. In J. Lloyd, V. Dahl, U. Furbach, M. Kerber, K.-K. Lau, C. Palamidessi, L. M. Pereira, Y. Sagiv, and P. J. Stuckey, editors, Proc. 1st Int. Conf. Computational Logic (CL2000), volume 1861 of Lecture Notes in Artificial Intelligence, pages 568-582, London, July 2000. Springer-Verlag.
[24] D. Kozen and F. Smith. Kleene algebra with tests: Completeness and decidability. In D. van Dalen and M. Bezem, editors, Proc. 10th Int. Workshop Computer Science Logic (CSL'96), volume 1258 of Lecture Notes in Computer Science, pages 244-259, Utrecht, The Netherlands, September 1996. Springer-Verlag.

[25] D. Kozen and W.-L. D. Tseng. The Böhm-Jacopini theorem is false, propositionally. Technical Report http://hdl.handle.net/1813/9478, Computing and Information Science, Cornell University, January 2008. Proc. 9th Int. Conf. Mathematics of Program Construction (MPC'08), July 2008, to appear.

[26] W. Peterson, T. Kasami, and N. Tokura. On the capabilities of while, repeat, and exit statements. Comm. Assoc. Comput. Mach., 16(8):503-512, 1973.

[27] J. Worthington. Automatic proof generation in kleene algebra. In R. Berghammer, B. Möller, and G. Struth, editors, 10th Int. Conf. Relational Methods in Computer Science (RelMiCS10) and 5th Int. Conf. Applications of Kleene Algebra (AKA5), volume 4988 of Lect. Notes in Computer Science, pages 382-396. Springer-Verlag, 2008. 